\title{
Coherent effects in laser spectroscopy of magnetic multilevel systems
}

\author{
Dieter Suter, Tilo Blasberg, and Harald Klepel \\ Institute for Quantum Electronics, ETH Zürich, $\mathrm{CH}-8093$ Zürich, and \\ Jürgen Mlynek \\ Department of Physics, Universität Konstanz, D-7750 Konstanz, Germany
}

\begin{abstract}
We present new experimental schemes for studying radiatively coupled atomic multilevel systems. These methods are aimed at creating order within the sublevel multiplets of complex multilevel systems and at extracting precise and detailed information about the order present in those systems. Time-resolved experiments provide not only static, but also dynamic information about these systems. Due to their high sensitivity and spatial selectivity, they may also be applied for magnetic resonance spectroscopy.
\end{abstract}

\section{INTRODUCTION}

The interaction between atomic systems and radiation is often described in terms of a model that treats the atom as a system of two energy levels interacting with a classical electromagnetic wave ${ }^{1}$. In reality, however, most atomic systems have considerably more complicated level structures. The ground state of atomic sodium, e.g., consists of eight energy levels grouped into two hyperfine multiplets (see figure 1). As is well known, this multiplicity leads to many interesting effects, like optical pumping, that cannot be explained with the simple two-level model. Since we Figure 1: Level structure of the Na ground are interested primarily in the dynamics of such multilevel sys- state.

tems coupled to optical fields, we use preferentially time-resolved experiments to study these systems, in contrast to the more conventional steady-state experiments ${ }^{2}$.

Our experimental investigations of these systems are performed with setups like the one shown in figure 2. The laser beam, which is derived from a single mode ring dye laser, is split into two parial beams, a pump beam with an intensity of the order of $100 \mathrm{~mW} / \mathrm{mm}^{2}$, and a probe beam with an intensity of the order of 1

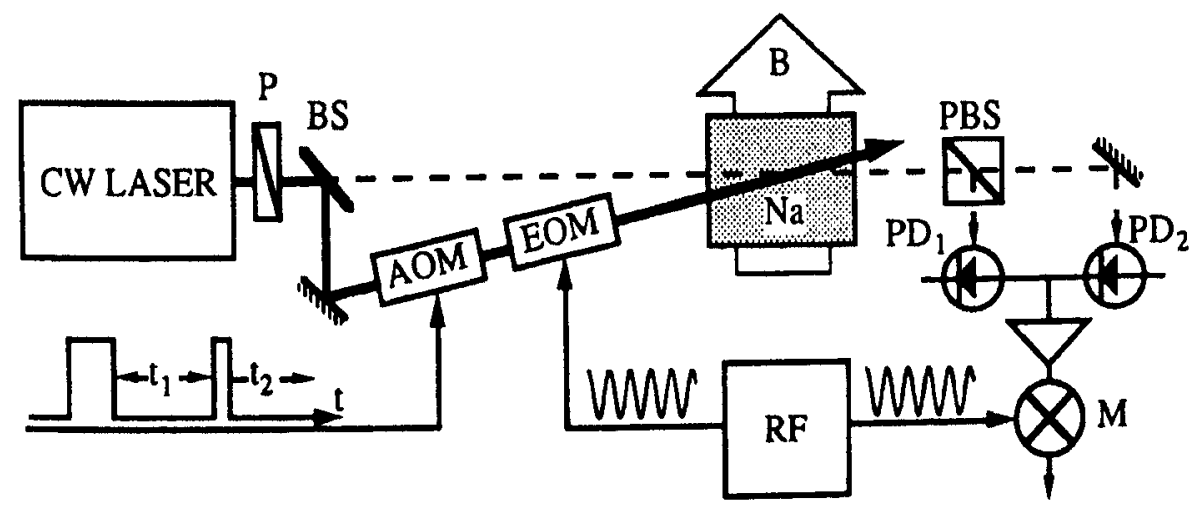

Figure 2: Experimental setup used for the investigation of atomic multilevel systems. $P$ $=$ polarizer, $\mathrm{BS}=$ beam split ter, $A O M=$ acoustooptic modulator, EOM = electrooptic modulator, $R F=$ radio-frequency synthesizer, $B=$ magnetic field, $\mathrm{PBS}=$ polarizing beamsplitter, $\mathrm{PD}_{1,2}=$ photodi . odes, $\mathbf{M}=$ mixer. 
$\mathrm{mW} / \mathrm{mm}^{2}$. The amplitude of the pump beam is controlled with an acoustooptic modulator (AOM) and its polarization can be modulated with an electrooptic modulator (EOM). For an efficient excitation of the atomic medium, which is located in a transverse magnetic field, the polarization of the pump laser beam is modulated between opposite circular polarizations. This procedure allows an efficient polarization of the atomic medium if the modulation frequency is close to the Larmor frequency ${ }^{3}$.

Pump and probe beam overlap in the sample region, where the metallic sodium is heated to a temperature of $\sim 140^{\circ} \mathrm{C}$ in the presence of $200 \mathrm{mbar}$ of Ar. The argon is used as a buffer gas to suppress the inhomogeneous Doppler broadening of the optical resonance line and to increase the time the atoms spend inside the laser beam. The probe laser beam, which is linearly polarized, is passed into a polarization-selective detector behind the sample cell. With the arrangement shown in figure 2 and the polarization of the incident probe beam rotated by $45^{\circ}$ with respect to the axis of the polarizing beam splitter, the detector produces a signal proportional to the angular momentum component of the $\mathrm{Na}$ atoms parallel to the laser beam. The signal which is recorded in such an experiment is modulated due to the Larmor precession of the magnetization in the external magnetic field. If the Larmor frequency exceeds a few hundred kilohertz, it is usually advantageous to partially eliminate this modulation by shifting the frequency into an experimentally more convenient region near zero. In our experiment, this is accomplished by passing the signal through a phase-sensitive detector which is locked to the modulation frequency that drives the EOM.

\section{COHERENT SUBLEVEL TRANSIENTS}

When a pump laser pulse is applied to the system, it excites coherences between the various sublevels of the electronic ground state. These coherences can then be detected via another Raman process with the probe laser beam. An example is shown in figure 3; here, a single, polarization-modulated laser pulse was applied to the sodium in a transverse magnetic field of $0.7 \mathrm{mT}$, corresponding to a Larmor frequency of $5 \mathrm{MHz}$. When the pump pulse is turned on, the system exhibits transient oscillations which are damped rapidly. After a time of the order of $10 \mu \mathrm{sec}$, it settles into a stationary state determined by the amplitude, frequency and polarization of the laser as well as by the strength and orientation of the magnetic field. After the end of the pulse, the magnetization present in the atomic system starts to precess around the magnetic field; this precession is observed as a free induction decay (FID) signal. Under the experimental conditions used for recording the data shown in figure 3 , the FID exhibits a series of equidist-

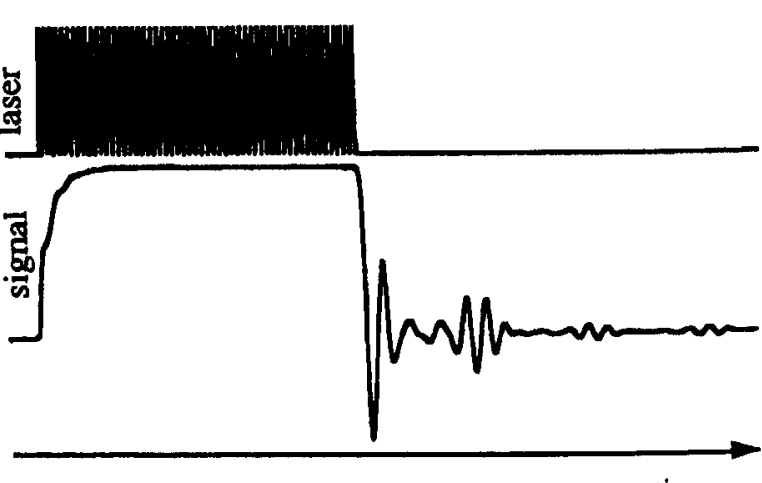

time

Figure 3: Response of the Na atoms to a pulse of polarization-modulated polarized light after phase sensitive detection at the modulation frequency.

ant features that resemble spin echoes. However, only a single pulse was applied to the system and their appearance does not depend on any inhomogeneities. A closer analysis shows, that these signal features cannot be explained in terms of the conventional "J=1/2 $\leftrightarrow \mathrm{J}^{\prime}=1 / 2$ " model ${ }^{4}$ that is conventionally used for the description of such experiments but does not take the nuclear spin $I=3 / 2$ of ${ }^{23} \mathrm{Na}$ into account.

The interpretation of the FID signal shown in figure 3 can be simplified considerably if it is Fourier transformed. The resulting spectrum, which is shown in figure 4, contains 6 distinct frequency components which can be assigned to Raman transitions between the various ground state sublevels. The resonance frequency of these sublevel transitions are shifted by different amounts due to the linear and quadratic electron Zeeman effect. As a result, the different transitions within each hyperfine multiplet are all non-equivalent. In addition, the nuclear Zeeman effect has opposite signs for the two hyperfine multiplets, making it possible to distinguish transitions within the $F=2$ hyperfine multiplet from those within the $F=1$ multiplet. The assign- 


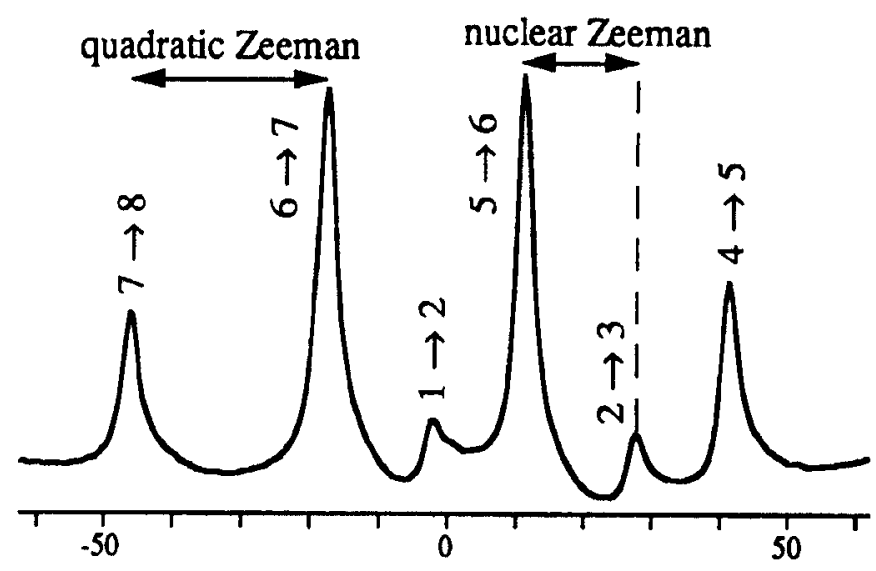

shift of resonance frequency $(\mathrm{kHz}) @ 5 \mathrm{MHz}$

ment of the various transitions is made in figure 4 in terms of the numbering of the energy levels introduced in figure 1. Clearly, the spectrum contains all six transitions between adjacent sublevels, i.e. between sublevels whose magnetic quantum numbers $m_{F}$ differ by $\left|\Delta m_{F}\right|=1$.

The absence of transitions between sublevels whose magnetic quantum numbers differ by $\left|\Delta \mathrm{m}_{\mathrm{F}}\right|>1$ can be understood by considering a hypothetical $\mathrm{Na}$ atom with vanishing nuclear spin. The electronic ground state consists then of only two sublevels and therefore only a single transition with $\left|\Delta \mathrm{m}_{\mathrm{J}}\right|=1$. In the limit of a weak hyperfine interaction, we expect a similar behaviour, i.e. to find only transitions with $\left|\Delta \mathrm{m}_{\mathrm{F}}\right|=1$. An exact calculation shows, that in the true $\mathrm{Na}$ ground state,

Figure 4: Fourier transform of the free induction decay signal shown in figure 3.

transitions with $\left|\Delta \mathrm{m}_{\mathrm{F}}\right|=2$ are allowed, but their amplitude varies roughly like $\Delta_{\mathrm{hf}} / \Gamma_{2}$, where $\Delta_{\mathrm{hf}}$ represents the hyperfine splitting (of the ground- as well as in the electronically excited state) and $\Gamma_{2}$ the width of the optical transition. In the case of a pressure-broadened optical transition, we expect therefore that signal contributions from higher order transitions $\left(\left|\Delta m_{F}\right|>1\right)$ should be negligible. In the case of the data shown in figure 4, e.g., the width of the $\mathrm{Na} \mathrm{D}_{1}$-transition was at least an order of magnitude larger than the hyperfine splitting of the excited state, so that the direct observation of transitions with $\left|\Delta m_{F}\right|=2$ would be rather difficult.

\section{OBSERVATION OF 'FORBIDDEN' RAMAN TRANSITIONS}

This and other selection rules can be circumvented by two-dimensional time-resolved spectroscopy. This technique is used extensively in the field of nuclear magnetic resonance (NMR) ${ }^{5}$ while applications to optical spectroscopy have not been demonstrated so far. The principle of the method is illustrated in figure 5: an initial laser pulse creates order in the form of population differences and coherences within the sublevel multiplets. Since this laser pulse may be arbitrarily strong, even coherences in 'forbidden' transitions, e.g. in transitions with $\left|\Delta \mathrm{m}_{\mathrm{F}}\right|=2$ can be excited via multiple Raman transitions. During the subsequent evolution period, these coherences are allowed to precess freely for a time $\mathrm{t}_{1}$. This free precession period 'labels' the coherences with a phasefactor $\exp \left(i \omega_{i k} t_{1}\right)$ which is specific for this transition ik. The subsequent mixing pulse causes an exchange of sublevel coherences between the various transitions. As a result, part of the coherence that evolved in transition ik may be transferred, together with the accumulated phase information, into a different transition. If this transition is Raman-active, it is therefore possible to observe the coherence and thereby measure the phase information associated with the forbidden Raman transition. The resulting signal depends then on the two independent time variables $t_{1}$ and $t_{2}$. By measuring the signal as a function of $t_{2}$
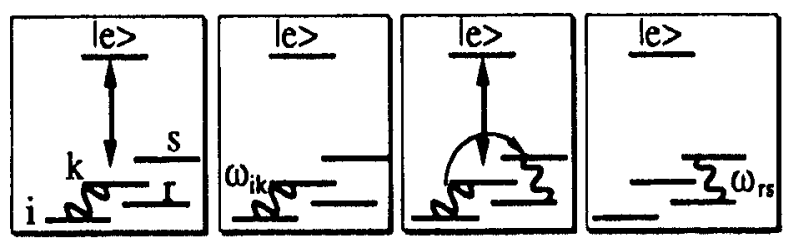

Preparation

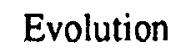

|Mixing! Detection

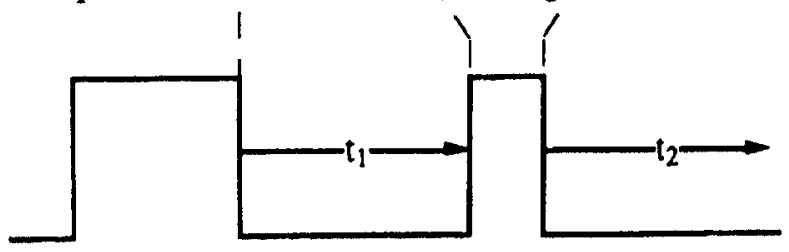

Figure 5: Schematic representation of the two-pulse scquence used for twodimensional spectroscopy and the effect on the atomic system. The individual periods are labclled with their conventional names. 


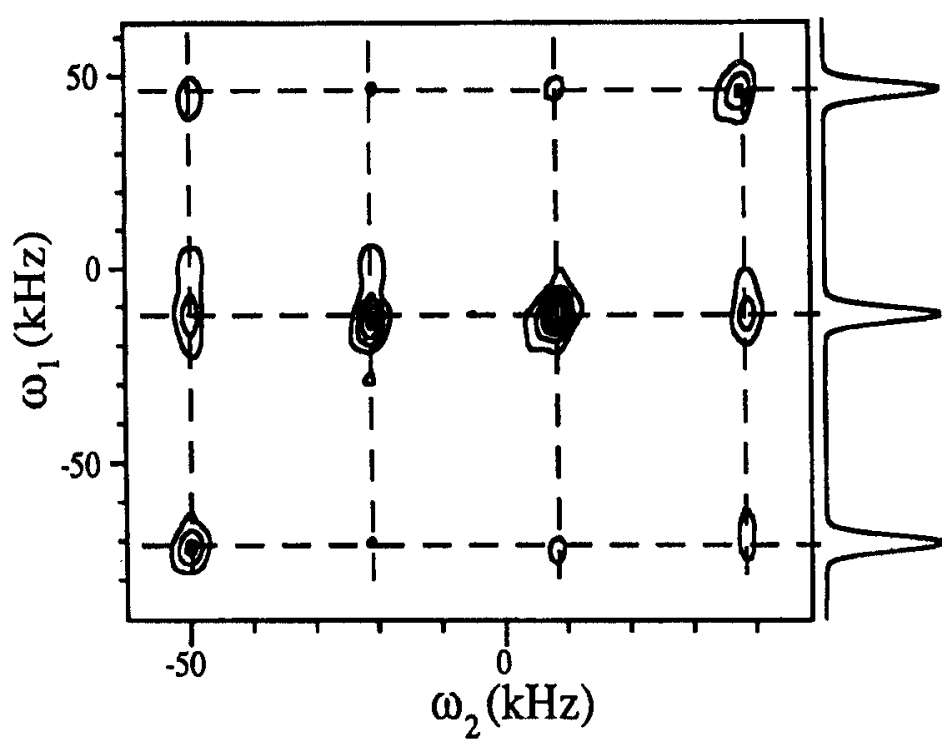

Figure 6: Example of a two dimensional spectrum demonstrating the possibility to use this method for observing 'forbidden' Raman transitions. The vertical axis represents the double quantum $\left(\left|\Delta \mathrm{m}_{\mathrm{F}}\right|=2\right)$ spectrum, while the horizontal axis corresponds to the single quantum spectrum. and repeating the experiment for a sequence of $t_{1}$ values, it is therefore possible to acquire a two-dimensional signal as a function of the two time variables. Fourier transformation in both dimensions leads then to a two dimensional spectrum.

In general, the initial pulse excites Zeeman coherences between all 8 sublevels of the $\mathrm{Na}$ ground state and the second pulse can transfer them into all observable single quantum transitions. The resulting two-dimensional spectrum contains therefore in general 52 distinct resonance lines. In many cases, it is therefore desirable to simplify the spectrum by suppressing unwanted resonances and concentrate on those in which one is specifically interested. Such an artificial 'selection rule' can be imposed by using the different effect that phase shifts of the modulation frequency have on the different types of coherences ${ }^{6}$.

In the example shown in figure 6 in contour plot representation, we have used this technique during the first pulse to excite coherences selectively in the $\left|\Delta m_{F}\right|=2$ transitions. In the spectrum, we expect therefore along the vertical axis only frequencies corresponding to transitions between next-nearest neighbours. The corresponding frequencies are indicated in the form of a one-dimensional spectrum to the right of the two-dimensional spectrum. After the second pulse, only the 'natural' selection rule $\left|\Delta m_{F}\right|=1$ is active; as a result, the same frequencies as in the one-dimensional spectrum appear along the (horizontal) $\omega_{2}$-axis. This spectrum shows therefore only those signals that are due to coherences which were created in $\left|\Delta m_{F}\right|=2$ transitions and subsequently transferred to $\left|\Delta \mathrm{m}_{\mathrm{f}}\right|=1$ transitions.

\section{OPTICALLY INDUCED COHERENCE TRANSFER DYNAMICS}

Clearly, this type of spectroscopy relies on the possibility to use laser pulses for transferring coherences between different sublevel transitions. This process, which was first observed in the context of laser-induced spin echoes ${ }^{6}$, has not been thoroughly investigated so far. A systematic investigation of the phenomenon is possible by recording two-dimensional spectra of the types shown in figure 6 as a function of the length of the second laser pulse ${ }^{7}$. This procedure allows a direct monitoring of the coherence transfer process. Figure 7 shows such a measurement of the transfer

Figure 7: Normalised intensity of cross peaks in two dimensional sublevel spectra of the $\mathrm{Na}$ ground state as a function of the length of the second pulse.

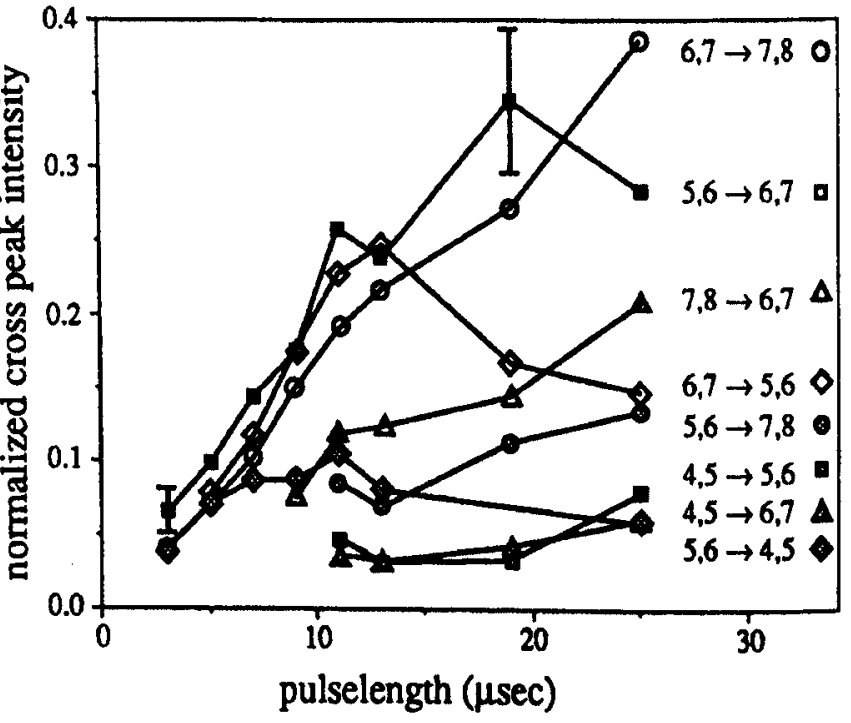


between single quantum sublevel transitions in the ground state of atomic sodium. The numbers on the right hand side, which refer to the energy levels as defined in figure 1, indicate to which particular transfer processes the symbols refer. While we have not yet evaluated the data quantitatively, these data show clearly that it is possible to observe the progress of the coherence transfer as the pulse length is increased. This procedure allows therefore for the first time a direct observation of laser-induced transfer of sublevel coherences.

\section{EVANESCENT WAVE SPECTROSCOPY}

The high sensitivity of the optical methods for the investigation of multilevel systems makes them also an attractive tool for the study of dilute or low-dimensional systems ${ }^{8}$, where the number of available atoms is relatively small. As an example for such an experiment, consider the setup shown in figure 8, which allows a selective investigation of $\mathrm{Na}$ atoms near a dielectric surface. The atomic system, again $\mathrm{Na}$ vapour, is contained in a glass cell which is covered by a glass prism. A pump laser beam propagates through the prism and the cell and optically pumps the atoms. The polarization of the atomic system leads to changes in the complex index of refraction which depends on the polarization of the light as well as on the polarization of the atomic medium. A probe laser beam, which is incident on the interface at an angle near the critical angle for total intemal reflection, is influenced by these changes in the refractive index as it is reflected from the interface. It is therefore pos-

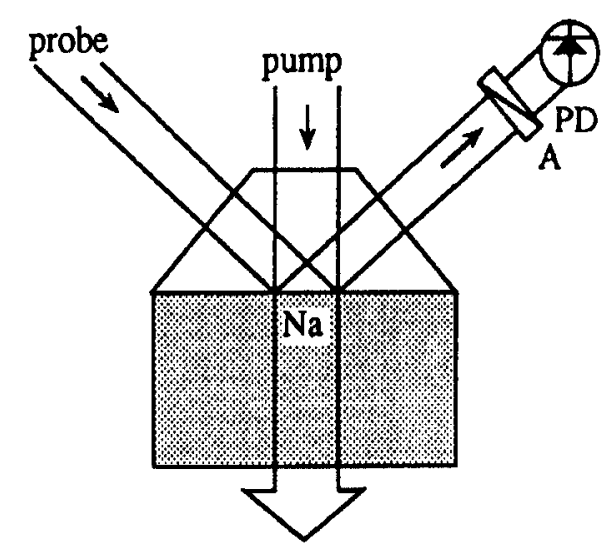

Figure 8: Principle of evanescent-wave spectroscopy of atomic multilevel systems. sible to obtain information about the atomic medium selectively from a layer near the interface whose thickness is of the order of an optical wavelength, by subjecting the reflected probe-beam to a polarization-selective detection. If a transverse magnetic field is applied and modulated optical pumping is used, the induced magnetic dipole moment exhibits Larmor precession and the resulting signal is modulated at the Larmor frequency. This makes it possible to use narrowband if detection schemes for increased sensitivity.

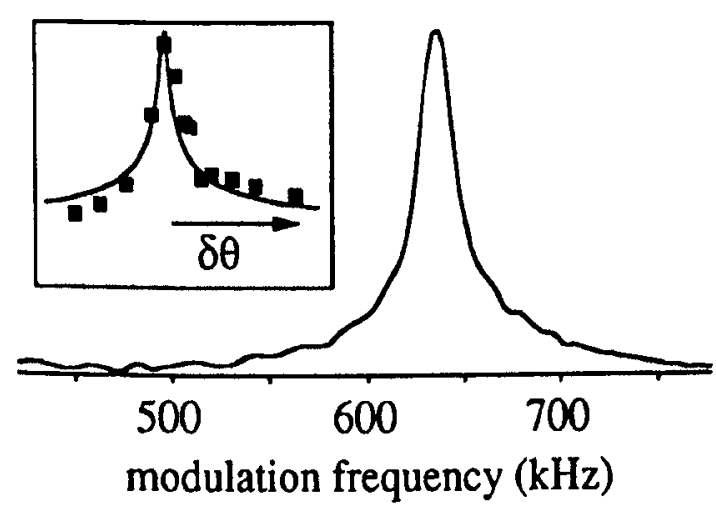

Figure 9: Example of a magnetic resonance spectrum of the $\mathrm{Na}$ ground state recorded with the setup of figure 8. The inset shows the dependence of the signal amplitude on the angle of incidence of the probe beam in a region close to the critical angle.
An example of a resulting sublevel spectrum is shown in figure 9 . For this experiment, the sample was placed in a magnetic field perpendicular to the pump beam as well as to the probe beam, parallel to the interface. By phase-sensitive detection of the signal as a function of the modulation frequency, it was therefore possible to record a magnetic resonance spectrum of the type shown in figure 9 . The inset shows the amplitude of the signal as a function of the angle of incidence of the probe beam in a region close to the critical angle for total intemal reflection. It shows clearly that the sensitivity of the method is maximised at the critical angle. The width of the curve is determined by the divergence of the probe laser beam which is slightly below $1 \mathrm{mrad}$ in our case. 


\section{SUMMARY AND CONCLUSION}

The techniques which we have presented here were developed for the purpose of obtaining precise and detailed information about the dynamics of coherences excited within the sublevel multiplets of realistic atomic multilevel systems. As an example, two-dimensional spectroscopy allows, for the first time, the observation of all possible transitions between Zeeman substates of the ground state of atomic sodium. The information which can be extracted about these systems is not only static, but also details of the sublevel dynamics can be observed, as we have demonstrated for the case of laser induced coherence transfer. The technique of two-dimensional time-resolved spectroscopy, which was applied for the first time in the domain of optical spectroscopy, has also a great potential for other forms of Raman spectra, as well as for the observation of electronic transitions. Possible extensions could make it possible to investigate excitation dynamics of electronic transitions or molecular dynamics.

Due to the high sensitivity of optical methods, the techniques developed here represent also an attractive alternative to classical magnetic resonance spectroscopy in cases where the sensitivity of the direct methods is not sufficient. As an example, we have demonstrated the possibility to observe magnetic resonance spectra in a quasi-twodimensional layer of an atomic gas close to a dielectric surface. Other possible applications may be found primarily in low-density solids or for the observation of magnetic resonance spectra of electronically excited states.

\section{ACKNOWLDEGMENTS}

We gratefully acknowledge financial support from the Swiss National Science Foundation.

\section{REFERENCES}

1. L. Allen and J.H. Eberly, Qptical Resenance and Two-Level Atoms, Dover Publications, New York, 1987.

2. A. Kastler, "Optical methods for studying hertzian resonances", Science. 158, pp 214-221, 1967.

3. Harald Klepel and Dieter Suter, "Transverse optical pumping with polarization-modulated light", Optics Commun., in print, 1992.

4. see, e.g., F. Mitschke, R. Desemo, W. Lange and J. Mlynek, "Magnetically induced optical self-pulsing in a nonlinear resonator", Phys. Rev. A 33 , pp 3219-3231, 1986.

5. R.R. Ernst, G. Bodenhausen and A. Wokaun, Principles of Nuclear Magnetic Resonance in One and Twe Dimensions, Oxford University Press, Oxford, 1987.

6. Dieter Suter, Martin Rosatzin, and Jürgen Mlynek, "Optically induced coherence transfer echoes between Zeeman substates", Phys. Rev. Lett. 67, pp 34-37, 1991.

7. Dieter Suter, Harald Klepel, and Jürgen Mlynek, "Time-resolved two-dimensional spectroscopy of optically driven atomic sublevel coherences", Phys. Rev. Lett. 67, pp 2001-2004, 1991.

8. Dieter Suter, Jérôme Äbersold, and Jürgen Mlynek, "Evanescent wave spectroscopy of sublevel resonances near a glass / vapour interface", Opt. Commun. 84, pp 269-274, 1991. 